... Markus Betschart, Kantonsarzt von St. Gallen

\title{
«lch bin Dienstleister für die anderen Ärzte»
}

\section{Daniel Lüthi}

Text und Bilder

danielluethi[at]gmx.ch
In den grauen Gestellen dieses bescheidenen Büros liegen Hunderte von farbigen Plastikmäppchen, sorgsam aufgeschichtet zu Dutzenden von kleinen Stapeln. Auf dem Pult liegen die aktuellen Themen und Fälle. Jedes Mäppchen ein Dossier. Markus Betschart nimmt eines nach dem andern vom Stoss und rezitiert eine Art Inhaltsverzeichnis seiner Tätigkeit: «Umfrage bei neuen Grundversorgern, Anerkennung eines ausländischen Doktortitels, kantonales EthikForum und Fachkommission Psychiatrie (Vorsitz), medizinische Richtlinien (Vernehmlassung), Arztzeugnis eines Arztes, der über 70 ist und weiterarbeiten will (prüfen), Pandemieplan (überarbeiten). «Ein Strauss verschiedener Arbeiten, nicht wahr?», kommentiert er und lächelt.

\section{Gesuche und Bewilligungen}

Geordnet nach Häufigkeit steht dies zuoberst auf der täglichen To-do-Liste: «Wir haben 15000 ausserkantonale Hospitalisationen pro Jahr, bei rund der Hälfte davon müssen wir Gesuche um Kostengutsprachen beurteilen.» Kriterium: «Ist eine Therapie in unserem Kanton verfügbar, kann ich ein Gesuch nicht bewilligen. Dies leider oft gegen den Willen eines Patienten oder seines Hausarztes.»

An zweiter Stelle steht das Thema «Sucht». Das Unterthema «Prävention» ist in der Verwaltung an einem anderen Ort angesiedelt, nämlich bei der Gesundheitsvorsorge, der Kantonsarzt kümmert sich in erster Linie um Methadon-Bewilligungen. «Wir haben im Kanton St. Gallen rund 900 Methadon-Pa-

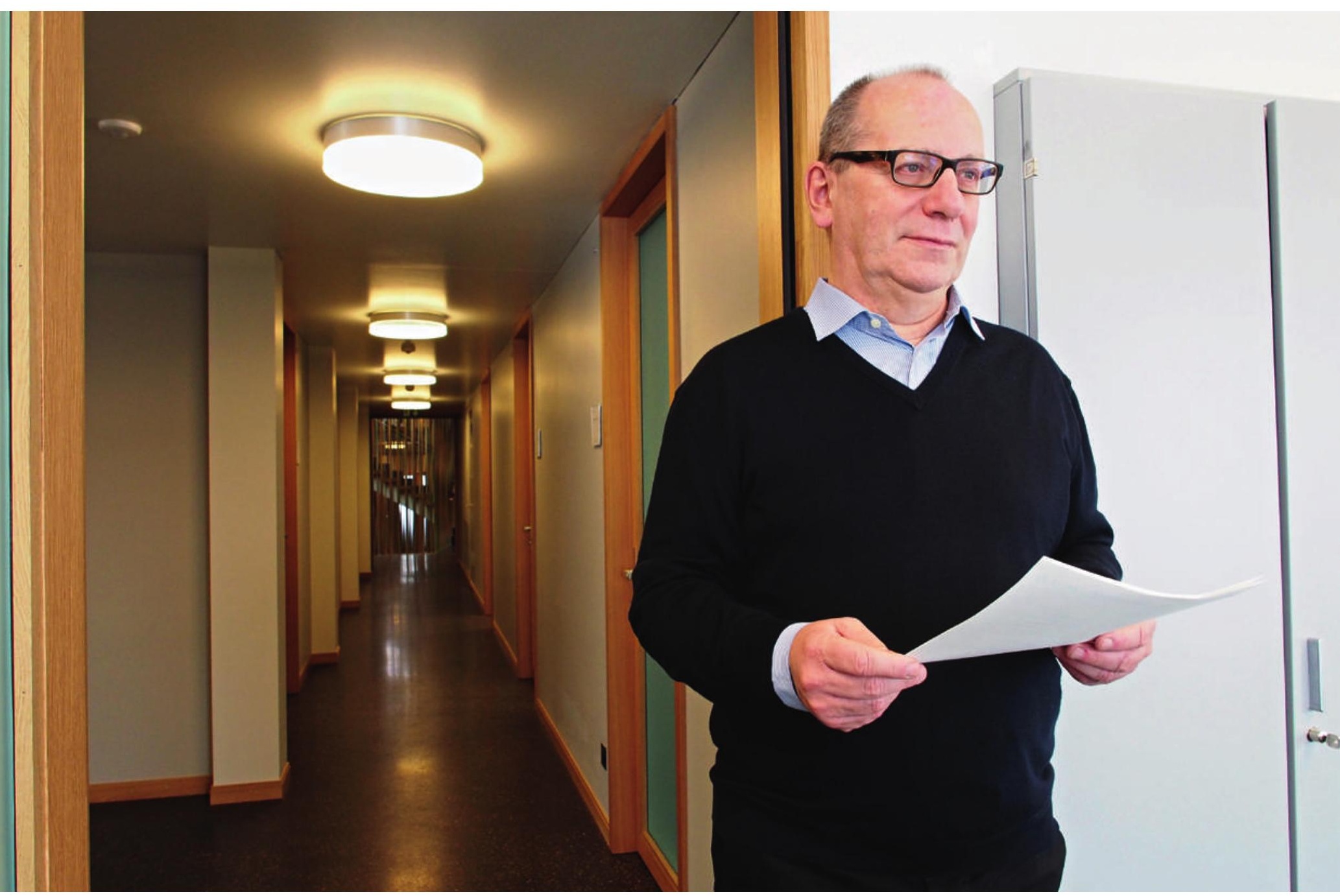


tienten. Es geht vor allem darum, den Konsum und den Handel auf der Gasse zu verhindern und den Beikonsum beispielsweise von Dormicum, Ritalin oder Sirdalud in den Griff zu kriegen. In solchen Fragen beraten wir auch die Hausärzte. Denn viele Drogensüchtige sind sehr fordernd, halten sich nicht an Vereinbarungen und machen Radau in der Arztpraxis. Deshalb versuchen wir zu erreichen, dass sich vermehrt unsere beiden psychiatrischen Kliniken um diese Patienten kümmern.» Im Übrigen weist Betschart darauf hin, dass die am meisten verbreitete Sucht eine andere ist: «Unser grösstes Problem, und es wird seit Jahrhunderten verdrängt, ist die Alkoholsucht.»

Drittes Hauptthema für den Kantonsarzt ist die Überwachung seiner Berufskollegen. "Wir können in den Praxen keine routinemässigen Kontrollen durchführen», sagt er dazu, «der Kantonsapotheker geht zu denen, die selber Medikamente verkaufen.» Schwarze Schafe? «Wird es schon geben. Erhalten wir Kenntnis davon, dann untersuchen wir die Klagen.» Im Kanton St. Gallen sind rund 1000 niedergelassene Ärztinnen und Ärzte registriert und mindestens gleich viele, die in Spitälern und Kliniken arbeiten. «Für die Zulassung der Spezialärzte bin ich auch zuständig.»

\section{«Unser grösstes Problem, und es wird seit}

\section{Jahrhunderten verdrängt, ist die Alkoholsucht.»}

Ein einziges Mal hat Betschart in den vergangenen bald zehn Jahren einem Arzt die Berufsausübungsbewilligung entziehen müssen. Zu den Gründen will er sich nicht äussern und ergänzt nur so viel: «Er hat dann trotzdem weitergemacht, wir mussten mit der Polizei hin und haben alles beschlagnahmt.» Ah ja, und einer hat die Bewilligung freiwillig abgegeben, «der hatte ein Alkoholproblem».

\section{Bürokratie als Herausforderung}

Bevor Betschart Beamter wurde bzw. in die Verwaltung wechselte, war er selber Arzt an der medizinischen Front. Dort wehren sich alle gegen die zunehmende Bürokratie - Betschart hat sie offensichtlich gesucht. Warum, fragt sich da. «Jede noch so interessante Tätigkeit ist mal jeden Tag gleich. Mit 55 fragte ich mich deshalb, ob ich noch zehn Jahre lang in der Intensivstation eines Spitals arbeiten will. Ich überlegte, ob ich noch Jus studieren soll, aber ich hatte den Mut nicht. Und dann kam - zum Glück, wie ich heute immer sage - ein neuer Chefarzt ins Spital, der mir Kompetenzen wegnehmen wollte. Es gab einen Konflikt, und gleichzeitig erschien das Inserat, in dem meine heutige Stelle ausgeschrieben war. Das war 2003. Ein schöner Zufall, der mir eigentlich ei-

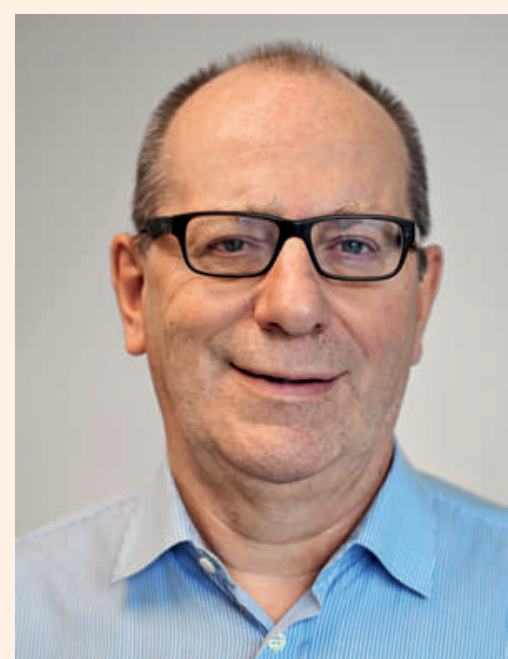

\section{Markus Betschart}

Dr. med. Markus Betschart wurde 1949 in Winterhur geboren, wo er aufwuchs und die Schulen besuchte. Medizinstudium und Staatsexamen (1975) absolvierte er an der Universität Zürich. Nach einem ersten Weiterbildungsjahr in der Anästhesieabteilung in Winterthur bildete er sich in Kardiologie und Nephrologie weiter. 1981 kehrte er in die Anästhesie zurück, und zwar am Kantonsspital Basel. 1990 machte er seinen Facharzttitel in Intensivmedizin. In diesem Spezialgebiet arbeitete er von 1988 bis 2003 am Kantonsspital St. Gallen als Leitender Arzt. 1999 schloss Betschart an der Fachhochschule für Wirtschaft in St. Gallen eine Weiterbildung zum «Executive Master of Health Service Administration» ab. Seit 1.1.2004 ist er Kantonsarzt von St. Gallen. Er ist zusätzlich unter anderem Vorstandsmitglied beim Nationalen Verein für Qualitätsentwicklung in Spitälern und Kliniken ANQ. Markus Betschart ist verheiratet und Vater von drei erwachsenen Kindern.

Er lebt mit seiner Familie in St. Gallen.

nen neuen Beruf bescherte: Ich bin Dienstleister für die anderen Ärzte. Ich will ihnen das Leben nicht erschweren, sondern erleichtern.»

Ein Arzt sucht vor allem die Nähe zum Patienten, den direkten Kontakt mit Menschen, die medizinische Hilfe benötigen. So stellt sich das der Laie vor, und so schildern es viele Ärztinnen und Ärzte. «Ich 
hatte eine schöne Zeit im Spital», antwortet Betschart, «aber die Patienten vermisse ich nicht.» Er hatte, wenn man's genau betrachtet, ja auch nie sehr viel direkt mit ihnen zu tun, das bestätigt er. Seine Patienten schliefen in der Regel, waren meistens nicht bei Bewusstsein. Das ist eine Eigenheit der beiden Spezialgebiete von Betschart, Anästhesie und Intensivmedizin. «Ja, vielleicht wollte ich gar nicht mit den Patienten reden - ich weiss es nicht», sinniert er. «Auf der IPS kamen vor allem die Angehörigen zu einem, das waren oft schwierige Gespräche, gerade wenn es um Organentnahmen ging. Auch mit den behandelnden Chirurgen musste man oft diskutieren, die wollten meistens mitreden. Die eigene Ohnmacht gab allerdings kaum einer zu, im Zweifelsfall wurde die Verantwortung oder Schuld dem IPS-Leiter zugeschoben.» Übrigens sei es entgegen der landläufigen Meinung keineswegs so, dass auf einer Intensivstation die Mehrzahl der Patienten sterbe, ergänzt Betschart, «95 Prozent überleben».

\section{Druck und Fehler}

Ein Spannungs- oder gar Konfliktfeld, wie er es selber erlebt hat, ist besonders anfällig für Fehler. Weil dies Betschart schon lange weiss, kümmert er sich seit langem besonders um das Thema "Qualitätsmanagement». «Wir waren in St. Gallen die Ersten im Land, die das «Critical Incident Reporting System CIRS` im Erwachsenenbereich einführten. Das war Mitte der 90er Jahre. Der Gedanke kam via Royal Air Force zu uns: Wenn jemand einen Fehler macht, soll er seinen Kolleginnen und Kollegen, aber auch den Vorgesetzten davon erzählen können, ohne Konsequenzen befürchten zu müssen - mit dem Ziel, dass der gleiche Fehler nicht mehr passiert. Je besser das Betriebsklima ist, umso mehr Meldungen gibt es.»

Beim Nationalen Verein für Qualitätsentwicklung in Spitälern und Kliniken ANQ ist Betschart als Kantonsvertreter im Vorstand. Hier geht es vor allem darum, wie die Qualität in Spitälern und Kliniken überhaupt gemessen werden kann. «Wir sagen in der Schweiz immer, die Qualität unserer Spitäler sei sehr gut - aber wir messen sie ja gar nicht», sagt er und ergänzt: «Es ist gar nicht so einfach, eine gute, flächendeckende und gleichzeitig möglichst günstige Messmethode zu finden. Möglichkeiten sind Patientenbefragungen, die Erhebung der Zahl von Rehospitalisationen und Reoperationen sowie von Infektionsraten.» Eine der bisherigen Erkenntnisse: «Je kleiner das Spital, desto zufriedener die Patienten. Das hat mit Übersichtlichkeit und Verbindlichkeit, mit der familiären Atmosphäre zu tun.» Eine andere: «Mehr Druck bedeutet mehr Fehler.» Kostendruck, Zeitdruck, Erwartungsdruck nehmen zu, mehr Rationalisierung und immer mehr Effizienz sind gefragt. Viele sagen, mit der Fallkostenpauschale sei dies noch schlimmer geworden. Wird es also mehr Fehler geben? «Ich weiss es nicht», antwortet Betschart.

\section{Hausärztesterben}

Beruflich sind Betschart die Grundversorger heute näher als die Spitalärzte. «Das Hausärztesterben ist in unserem Kanton heute noch kein Problem», kommentiert er, «aber etwa die Hälfte der Grundversorger ist über 55-jährig. Das Problem kommt also auf uns zu.» Und was macht er dagegen? Praxisbewilligungen ausstellen zum Beispiel. «Letztes Jahr waren es rund 50. 34 davon gingen in Gesundheitszentren oder Gruppenpraxen, das ist wohl die Zukunft. Übrigens: Von den 50 neuen Ärzten waren nur 18 Schweizer.» Dazu hat der Kanton schon 2007 ein Förderungsprogramm für die Hausarztmedizin geschaffen bzw. für eine Million Franken 10 Stellen. «Angehende Hausärzte können so viermal ein je sechsmonatiges Praktikum in einer Spezialdisziplin machen, zum Beispiel in der Kindermedizin oder der Psychosomatik. Und sie haben einen Tutor, der mit ihnen ihre Laufbahn plant.»

\section{«Mehr Druck bedeutet mehr Fehler.»}

Quote auf Betscharts berufliche Laufbahn neigt sich dem Ende zu. Trotzdem denkt er noch nicht ans Aufhören, im Gegenteil. Für die kahlen Wände in seinem Büro will er nächstens im Amt für Kultur nach dekorativen Bildern Ausschau halten, «etwas Modernes» wünscht er sich. «Ich hätte ja mit 63 schon gehen können», kommentiert er in gewohnt bedächtiger Manier, «aber so lange es mir gefällt, bleibe ich - obschon die Arbeitsbelastung ähnlich ist wie damals auf der IPS, bloss anderer Art. 50 bis 60 Stunden pro Woche arbeite ich inklusive Abendsitzungen mindestens. Hobbys habe ich, wie viele andere Ärzte auch, praktisch keine. Ich schiebe also keineswegs eine ruhige Kugel, wie viele vermuten.»

Am Ende jeden Monats stellt die Schweizerische Ärztezeitung eine Persönlichkeit vor, die sich im Gesundheitswesen engagiert. Im April schildert Daniel Lüthi seine Begegnung mit Sarah Bär, Medizinstudentin. 